\title{
Fibrilación ventricular inducida por electrocauterización en cirugía videolaparoscópica
}

\author{
Pirez Gornatti V. ${ }^{1}$, Campos Silveira C. ${ }^{1}$, Faleiro Rodrigues L. ${ }^{1}$, Coelho Barroso F. ${ }^{1}$, Mateus Serzedo PS. ${ }^{1}$, Criscuolo \\ Miksche L. ${ }^{1}$ \\ 1 Clínica de Anestesiología de Ribeirão Preto, Ribeirão Preto, Brasil. \\ Introducción: La cirugía videolaparoscópica se caracteriza por ser una intervención mínimamente invasiva. Utiliza la \\ electrocauterización, la cual se rige por un conjunto de leyes físicas que determinan el camino que seguirá la corri- \\ ente. Se han reportado eventos adversos cuando se utiliza la electrocoagulación, especialmente al utilizar el sistema \\ monopolar.
}

Caso clínico: Hombre, 38 años, PS1, reflujo gastroesofágico y esofagitis con indicación de cardioplastía. Exámenes preoperatorios sin alteraciones. Técnica anestésica, venosa total. En el intraoperatorio, durante la disección del esófago abdominal, en la región subdiafragmática izquierda, con electrocauterización monopolar en coagulación 40V, el paciente evoluciono en fibrilación ventricular. El procedimiento fue interrumpido e iniciadas las maniobras de resucitación cardiopulmonar seguidas de desfibrilación con $200 \mathrm{~J}$, se logró el regreso de la circulación espontanea. El procedimiento fue abortado, el paciente encaminado a la unidad de cuidados intensivos, lúcido y estable. Luego de 24 horas en observación, recibió alta.

Discusión: La electrocauterización tiene la capacidad de generar efecto de corte o coagulación y puede ser utilizada en sistema monopolar o bipolar. El monopolar es el más utilizado, en este, se utiliza un electrodo activo para conducir la corriente, y un electrodo de dispersión, la placa. Bastante tejido está expuesto a la electricidad, ofreciendo mayores riesgos. En el sistema bipolar, los electrodos están separados por una pequeña distancia, de 1 a $3 \mathrm{~mm}$, limitando el flujo de la corriente. Las lesiones ocurren con mayor frecuencia en donde está situada la placa, así como, en sitios que funcionan como vía alternativa para la dispersión de electricidad. La región del corazón, por ejemplo. El miocardio es sensible a frecuencias entre $30 \mathrm{~Hz}$ y $110 \mathrm{~Hz}$. La corriente puede producir un arco entre el tejido y el electrodo, ocurriendo cambio de alta a baja frecuencia $(50-60 \mathrm{~Hz})$, lo que puede inducir fibrilación ventricular. Además, este efecto es facilitado por la baja resistencia del tejido miocárdico debido su alta vascularización.

Conclusión: Es fundamental seguir las normas de seguridad. El correcto posicionamiento de los electrodos. Evitar el contacto con objetos metálicos. Utilizar la potencia más baja posible para lograr el efecto deseado. La ubicación de la placa dispersiva debe estar lo más cerca del sitio quirúrgico, en una zona bien vascularizada y con mayor masa muscular, para una mejor conducción eléctrica. Cuando utilizamos el sistema monopolar, asegurarse de que la distancia entre los electrodos activos y dispersivos sea lo más corta posible y no cruce el eje cardíaco.

https://doi.org/10.25237/congresoclasa2019.43 